\title{
Reduction of Grating Lobes Due to Dividing the Array into Uniform Subarrays Using Genetic Algorithm
}

\author{
A.A. Omer ${ }^{*}$, and A.A. Mitkees ${ }^{\dagger}$
}

\begin{abstract}
The total cost of the large arrays can be solved by dividing the array into contiguous subarrays with amplitude and time delay devices at subarray level. This cause appearance of grating lobes when the beam has been steered to small angle away from broadside named grating lobes due to uniform subarrays. These grating lobes caused by the periodicity and the larger spacing between subarrays. If there is a need to steer the main beam at angle that will be small (limited scan), some methods are used to reduce these grating lobes. These methods will be optimized using genetic algorithm implemented using MATLAB. Two approaches used to disrupt the periodicity in the array, so reduction of the grating lobes can be achieved. Three parameters are optimized simultaneously, the amplitude weighting at the subarray ports; the number of elements per subarray and the spacing between elements. The results show that the two approaches reduce the grating lobes upto $-9.8 \mathrm{~dB}$ for the first approach and $-14.6 \mathrm{~dB}$ for the second approach, when steered over small-scan angles, up to $15^{\circ}$ from broadside, after this range grating lobe will appears again and cannot be reduced.
\end{abstract}

Keywords: Uniform subarrays, grating lobes, genetic algorithm, nonuniform subarrays.

\section{Introduction}

Large antenna arrays are partitioned into subarrays in order to reduce cost through common use of components or mass production of identical subarrays [1]. Typically, time delay units for beam steering large phased arrays are placed at the output of a subarray due to the high cost of placing a time delay unit at each element. Placing amplitude weights and time delay unit at the subarray ports rather than the elements creates grating lobes [2].

One way to eliminate the grating lobes is to create overlapped subarrays using Butler matrix or lens feeds. Some examples of overlapped constrained feed networks are found in [2]. The elements in the subarray can be uniformly weighted or have an amplitude taper that is the same for all subarrays [3]. Other approaches have tried randomizing the number of elements within a given subarray [4], rotating the subarray orientation [5], and nonuniformly spacing the elements within the subarrays [6]. Throw this paper two approaches are used to disrupt the periodicity in the array.

\footnotetext{
* Lecturer Assistant, BHIT, Benha University, atelep82@yahoo.com .

${ }^{\dagger}$ Egyptian Armed Forces, Egypt, aamitkees@yahoo.com . 


\section{The Array Divided Into Subarrays}

If the array has been divided into $M$ subarrays with $N$ elements in each subarray and amplitude taper $w(m)$ at subarray level. The array factor will be

$$
S_{a}(\theta)=\sum_{m=1}^{M} w(m) \sum_{n=1}^{N} e^{j \kappa_{o}((m-1) N+n) d \sin (\theta)}
$$

where $w(m)$ is the amplitude weight at subarray $m, M$ is the number of subarrays, $N$ is the number of elements per subarray and $d$ is the uniform distance between elements.

In the previous equation the main beam will point to the broadside direction. If there was a need to scan the beam, a time delay units should be inserted. These time delay units are placed at the output of subarrays due to the high cost of placing a time delay unit at each element as shown in figure 1 . The array factor will be

$$
S_{a}(\theta)=\sum_{m=1}^{M} w(m) e^{j \kappa_{o}(m-1) N d\left[\sin (\theta)-\sin \left(\theta_{o}\right)\right]} \cdot \sum_{n=1}^{N} e^{j \kappa_{o} n d \sin (\theta)}
$$

where $w(m)$ is the amplitude weight at subarray $m, M$ is the number of subarrays, $N$ is the number of elements per subarray, $d$ is the uniform distance between elements and $\theta 0$ is the pointing angle. Placing amplitude and phase weighting at the subarray outputs alone causes grating lobes in the array factor due to the periodicity and the larger spacing between subarrays.

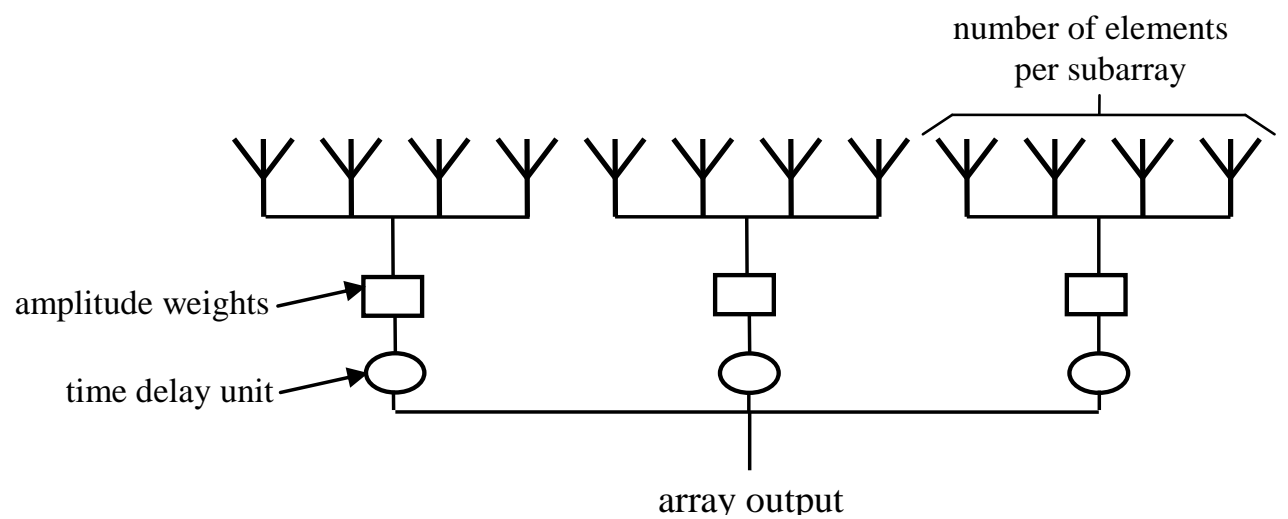

Fig. 1 The array divided into contiguous subarrays with time delay unit to steer the main beam at any angle.

In this configuration, if the beam steered away from broadside, grating lobes will appeared as shown in figure 2 . These grating lobes will be reduced using three techniques:

1. Optimizing the subarray amplitude weights, the amplitude weightings at the subarray outputs are optimized to reduce the highest SLL/GL.

2. Optimizing the subarray sizes, the randomizing of the number of elements at each subarray disrupts the array periodicity resulting in the reduction of the peak SLL/GL.

3. Optimizing the spacing between the elements, the nonuniform spacing between elements also disrupts the array periodicity and consequently reduces the peak SLL/GL. 
These techniques will make reduction of grating lobes up to $-14 \mathrm{~dB}$ for steering to angle $15^{\circ}$ right and left broadside direction as shown in figure 3 , so it will be named limited scan.

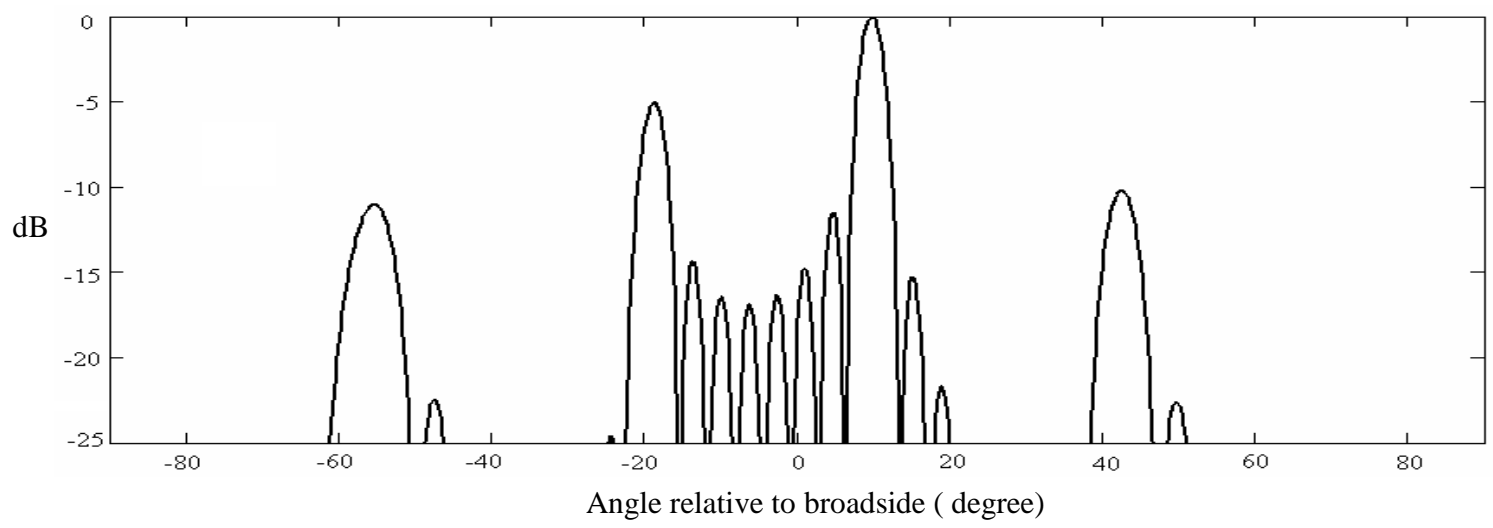

Fig. 2 Power radiation pattern of the array factor of a linear 32-element phased array antenna divided into 8-subarrays with 4-elements in each subarray with uniform element spacings $=\lambda / 2$, phased for beam pointing at $\theta_{0}=10^{\circ}$ from broadside.

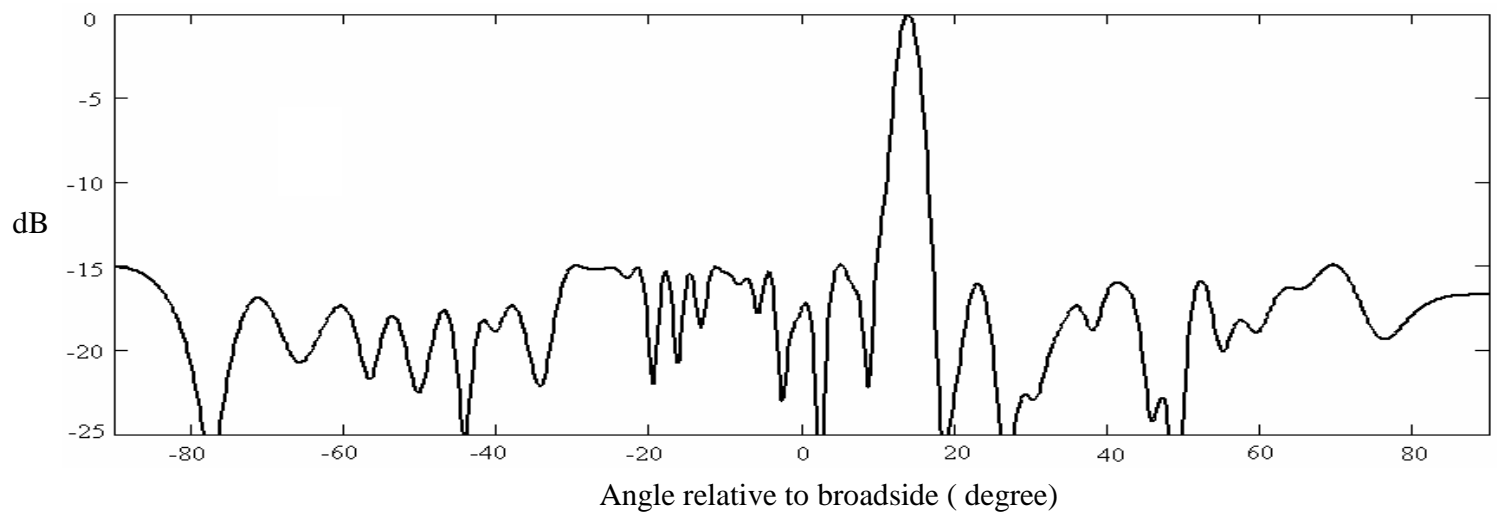

Fig. 3 Power radiation pattern of the array factor of a linear 64-element phased array antenna divided into subarrays with optimized amplitudes at subarray outputs, number of elements per subarray and spacing between elements, phased for beam pointing at $\theta_{0}=15^{\circ}$ from broadside.

\section{Genetic Algorithm}

A genetic algorithm is an iterative optimization algorithm prepared to emulate the processes of evolutionary biology. A block diagram for simple genetic algorithm is shown in figure 4 [7]. The genetic algorithm operators are crossover and mutation, our genetic algorithm use one point crossover and $20 \%$ mutation rate. This paper use population decimation as a selection strategy. The fitness of each individual will be evaluated using the fitness function which is defined by the maximum relative sidelobe level. The algorithm minimizes the fitness of the population. 


\section{Optimization Results}

\subsection{Optimizing the subarray amplitude weights and the number of elements per subarray}

By dividing the array into subarrays of unequal sizes and amplitude weighting at the subarray ports, reduction of grating lobes due to uniform subarray can be achieved, as shown in figure 5 . The total number of elements in the array is given by

$$
N_{T}=\sum_{m=1}^{M} N(m)
$$

where $N_{T}$ is the total number of elements in the array, $M$ is the number of subarrays and $N(m)$ is the number of elements in subarray $m$.

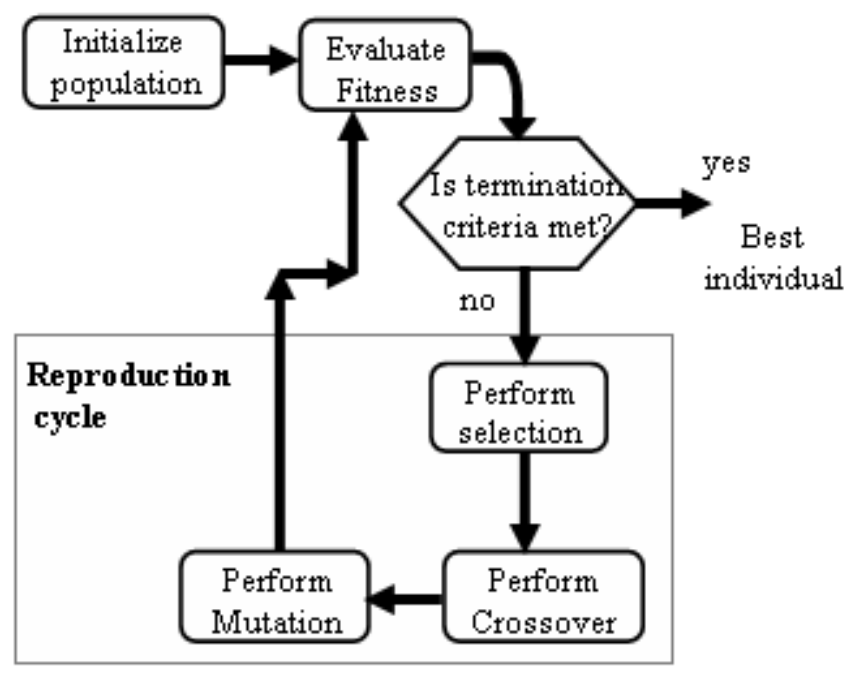

Fig. 4 Block diagram of a simple genetic algorithm optimizer

The fitness function used in genetic algorithm program will be

$$
F F=\max \left(\frac{\sum_{m=1}^{M} w(m) e^{j \kappa_{o}\left[\left(\sum_{i=1}^{m} N(i)\right)-N(m)\right] d\left[\sin (\theta)-\sin \left(\theta_{o}\right)\right]} \sum_{n=1}^{N(m)} e^{j \kappa_{o} n d \sin (\theta)}}{\max (A F)}\right)
$$

where $w(m)$ is the amplitude weight at subarray $m$ (first parameter that will be optimized), $M$ is the number of subarrays, $N(m)$ is the number of elements of subarray $m$ (second parameter that will be optimized), $N(i)$ is the number of elements of subarray $i, d$ is the uniform distance between elements will be $0.5 \lambda$ and $\theta_{\mathrm{o}}$ angle that the array is steered from broadside. 


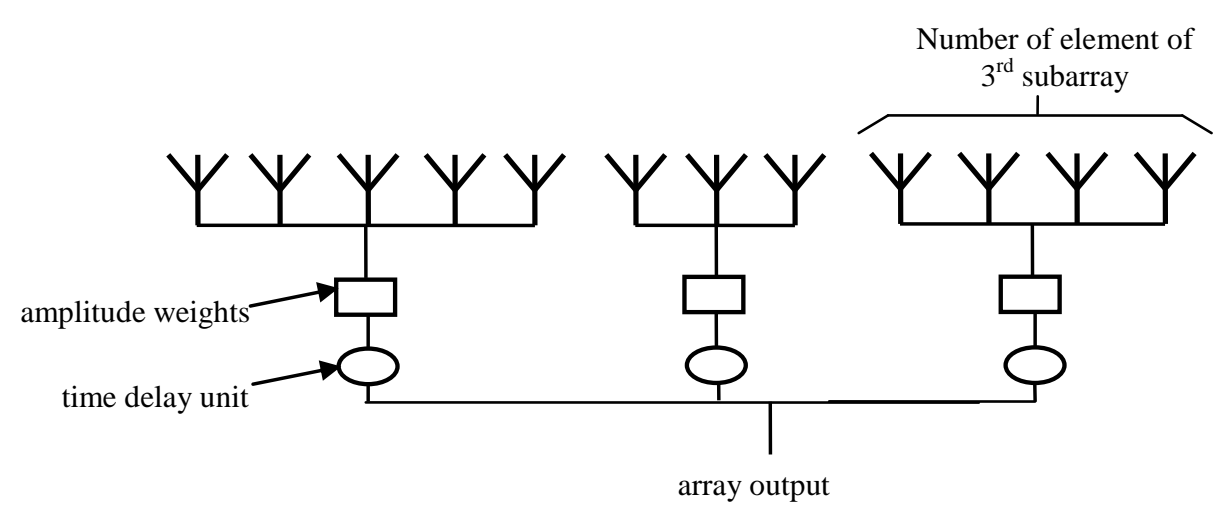

Fig.5 An array divided into subarrays of unequal size with amplitude weights and time delay units at subarray level.

Each subarray starts with minimum number of elements $\mathrm{N}_{\mathrm{emin}}$ that is allowed in subarrays, the remaining elements $\left[N_{T}-\left(M \times \mathrm{N}_{\mathrm{emin}}\right)\right]$ are distributed among the subarrays. Selecting $\mathrm{N}_{\mathrm{emin}}$ is arbitrary unless certain design constrain exist. In order to take advantage of the efficiencies offered by subarrays, take $\mathrm{N}_{\mathrm{emin}} \geq 2$. If this minimum number of elements is changed, the reduction will be affected. Amplitude should be assigned for each subarray.

For example, if an array of 64 elements divided into 16 subarrays of equal number of element per subarray steered to $14^{\circ}$ from broadside, grating lobes will appears. By applying genetic algorithm for amplitudes at subarray level and number of elements of subarrays for minimum number of elements per subarray equal to $2,-9.8 \mathrm{~dB}$ reductions is achieved as shown in figure 6 . The distribution of number of elements and subarray weights is shown in figure 7.

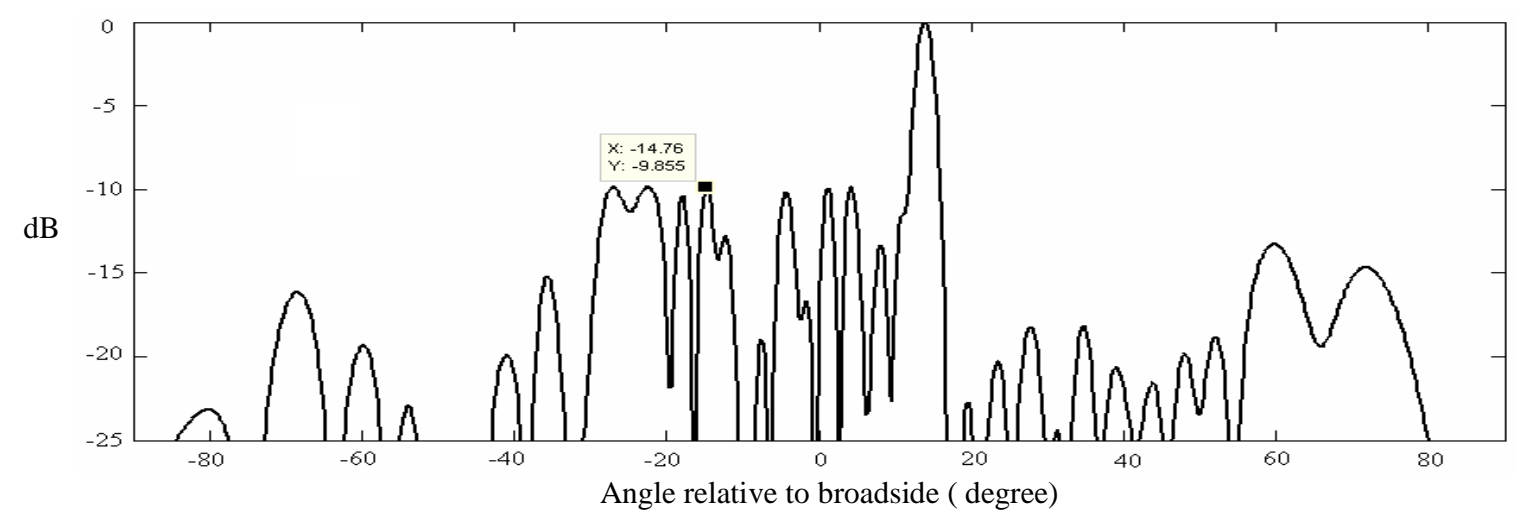

Fig. 6 Power radiation pattern of the array factor of optimized array divided into subarrays with unequal size and amplitude taper at subarray level.

\subsection{Optimizing the subarray amplitude weights, number of elements per subarray and the spacing between elements}

A combined approach to disrupt the periodicity in the array is proposed to reduce the grating lobes. In this approach, three measures are simultaneously used. They are: (1) the optimized amplitude weighting at the subarray ports; (2) optimize the number of elements per subarray; and (3) optimize the spacing between elements, more reduction can be achieved. Instead of uniform spacing of $0.5 \lambda$ between elements, it will be nonuniform spacing, in this case the maximum disturbance is $0.5 \lambda$, constrains for minimum number of elements per subarray will be as previous case. The fitness function is 


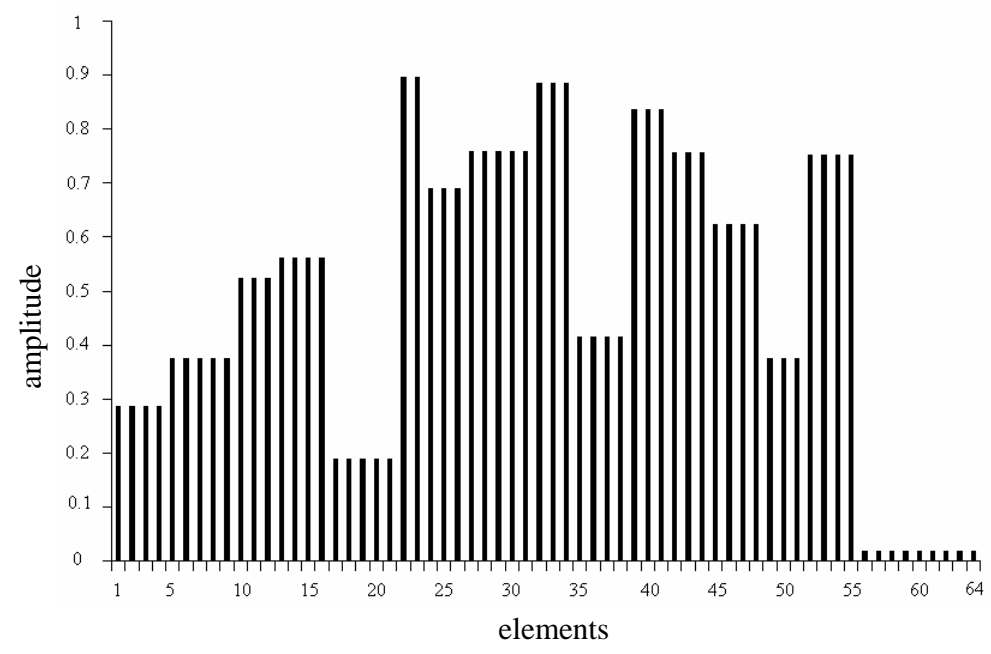

Fig. 7 The number of elements in the subarrays and the subarray weights.

$$
F F=\max \left(\frac{\sum_{m=1}^{M} w(m) e^{j \kappa_{o} d_{m}\left[\sin (\theta)-\sin \left(\theta_{o}\right)\right]} \sum_{n=1}^{N(m)} e^{j \kappa_{o} d_{n} \sin (\theta)}}{\max (A F)}\right)
$$

where $w(m)$ is the amplitude weight at subarray $m$ (first parameter that will be optimized), $M$ is the number of subarrays, $N(m)$ is the number of elements of subarray $m$ (second parameter that will be optimized), $d_{m}$ is the distance between subarrays, $d_{n}$ is the distance between elements in subarray (third parameter that will be optimized) and $\theta$ o angle that the array is steered from broadside.

If spacing between elements are optimized then spacing between subarrays will be changed, that must be calculated through the program, this spacing depend on number of elements in that subarray and spacing between them. A reduction of $-14.6 \mathrm{~dB}$ can be achieved. The power radiation pattern is shown in figure 8 . The distribution of elements in subarrays with amplitude weighting is shown in figure 9.

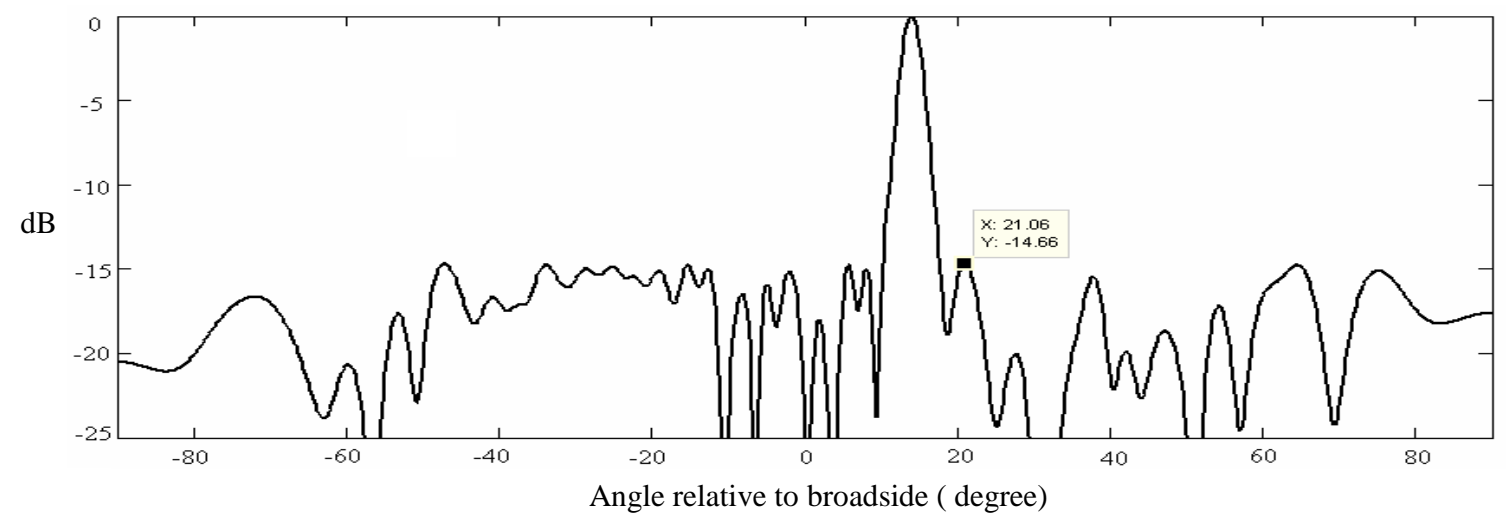

Fig. 8 Power radiation pattern of the array factor of optimized array divided into subarrays with unequal size, amplitude taper at subarray level and nonuniform spacing between elements. 


\section{Conclusions}

For linear array divided into uniform subarray with amplitude and phase weights at the subarray ports, two approaches used to disrupt the periodicity in the array, so reduction of the grating lobes can be achieved.

The first approach, two parameters are optimized simultaneously, they are: (1) the amplitude weighting at the subarray ports; and (2) the number of elements per subarray.

The second approach, three parameters are optimized simultaneously, they are: (1) the amplitude weighting at the subarray ports; (2) the number of elements per subarray; and (3) the spacing between elements.

The results show that the two approaches reduce the grating lobes upto $-9.8 \mathrm{~dB}$ for the first approach and $-14.6 \mathrm{~dB}$ for the second approach, when steered over small-scan angles, up to $\pm 15^{\circ}$ from broadside, after this range grating lobe will appears again and can't be reduced.

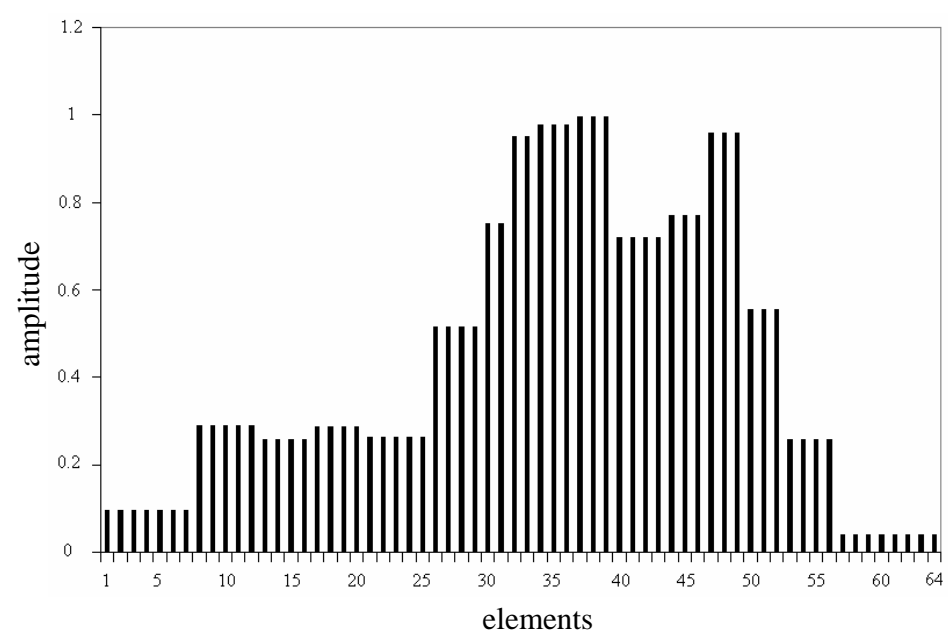

Fig. 9 The number of elements in the subarrays and the subarray weights.

\section{References}

[1] R. L. Haupt, "Optimized Weighting of Uniform Subarrays of Unequal Sizes," IEEE Trans. Antennas Propag., vol. 53, pp. 1207-1210, Apr. 2007.

[2] R. J. Mailloux, Phased Array Antenna Handbook. Boston, MA: Artech House, 2005.

[3] R. L. Haupt, "Reducing Grating Lobes due to Subarray Amplitude Tapering," IEEE Trans. Antennas Propag., vol. 33, pp. 846-850, Aug. 1985.

[4] A. P. Goffer, M. Kam, and P. R. Herczfeld, "Design of Phased Arrays in Terms of Random Subarrays," IEEE Trans. Antennas Propag., vol. 42, pp. 820-826, Jun. 1994.

[5] P. S. Hall and M. S. Smith, "Sequentially Rotated Arrays With Reduced Sidelobe Levels," IEE Proc. Microw. Ant. Prop., vol. 141, pp. 321-325,Aug. 1994.

[6] N. Toyama, "Aperiodic Array Consisting of Subarrays for Use in Small Mobile Earth Stations,” IEEE Trans. Antennas Propag., vol. 53, pp.2004-2010, Jun. 2005.

[7] J. M. Johnson, Y. R. Samii, "Genetic Algorithms in Engineering Electromagnetics," IEEE Antennas Propagat. Mag., vol. 39, pp. 7-21, Aug. 1997. 